\title{
2. Valence, prédicat, préposition et la notion d'adjet
}

\section{Michael Herslund}

\section{(2) OpenEdition}

\section{Journals}

Édition électronique

URL : https://journals.openedition.org/ml/568

DOI : $10.4000 / \mathrm{ml} .568$

ISSN : 2274-0511

Éditeur

Association Modèles linguistiques

\section{Édition imprimée}

Date de publication : 1 juillet 2006

Pagination : 15-24

Référence électronique

Michael Herslund, «2. Valence, prédicat, préposition et la notion d'adjet », Modèles linguistiques [En ligne], 54 | 2006, mis en ligne le 01 octobre 2015, consulté le 01 juillet 2021. URL : http://

journals.openedition.org/ml/568; DOI : https://doi.org/10.4000/ml.568

Ce document a été généré automatiquement le 1 juillet 2021.

(C) Modèles Linguistiques 


\title{
2. Valence, prédicat, préposition et la notion d'adjet
}

\author{
Michael Herslund
}

\section{Introduction}

1 L'analyse de la proposition ne se réduit pas à la simple attribution d'étiquettes - telles que sujet, objet, complément adverbial, etc. - aux unités identifiées. Au contraire, il s'agit de dégager le réseau de relations qui constituent la proposition. Le noyau de ce réseau est constitué par un prédicat, réalisé par un verbe le plus souvent, et c'est la valence de ce noyau qui détermine la structure de la proposition par les arguments sélectionnés et les relations grammaticales attribuées à ces arguments, réalisés le plus souvent par des syntagmes nominaux. Certains arguments sont pourtant réalisés par des syntagmes prépositionnels. Le but du présent article est d'examiner le statut de ces arguments, et le rôle de la préposition, dans le réseau construit autour du verbe.

\section{Théorie de la valence et la distinction entre actants et circonstants}

2 Le prédicat (le verbe) exprime un réseau de relations entre des entités qui jouent chacune un rôle particulier dans la situation en question. Par conséquent, il détermine la présence de certains compléments, c'est-à-dire la réalisation grammaticale des arguments qui entrent dans le réseau de relations établi par le prédicat, donc ceux qui saturent la valence du prédicat : les actants selon la terminologie de L. Tesnière (1959). Cela revient à dire qu'un actant se définit comme un complément prévu par le contenu sémantique du prédicat. C'est ainsi qu'un verbe tel que donner décrit une situation avec des relations entre trois participants - les actants du verbe - à savoir celui qui donne, quelque chose donné et celui qui reçoit :

3 [1] L'été, Julie donne toujours ses vêtements d'hiver aux pauvres. 
4 Les autres membres de la proposition, ceux qui ne saturent pas la valence du prédicat, sont des circonstants, qui n'entrent pas dans le réseau de relations établi, comme par exemple l'été de l'exemple (1) : ce n'est pas le verbe donner qui établit la relation entre ce complément et le reste de la proposition. Comme on le sait, la distinction de L. Tesnière entre actants et circonstants est théoriquement facile et évidente, mais en pratique, on est souvent dans le doute devant des cas précis. Les cas douteux sont pour la plupart des compléments locaux prépositionnels (cf. Leeman 1998). Sans trop entrer dans les détails, on peut dire que la différence décisive est la suivante : ou bien c'est le prédicat qui établit la relation identifiée entre un de ses arguments et l'argument local, ou bien l'argument local ne désigne que la scène où a lieu la situation décrite. On trouve donc une différence nette entre les deux exemples suivants :

5 [2] a. Julie entre dans la piscine.

b. Julie nage / se maquille / lit Balzac dans la piscine.

6 Alors que dans (2a) c'est le prédicat entrer qui établit la relation entre Julie et la piscine, dans $(2 b)$ le complément dans la piscine ne désigne que la scène où se déroulent les activités dénotées par les prédicats nager, se maquiller, lire Balzac. On dira donc que dans le premier cas, dans la piscine est un actant, dans le second, un circonstant, malgré le fait qu'il s'agit dans les deux cas de matériaux grammaticaux identiques.

\section{Les actants}

\subsection{L'actant fondamental}

7 Or, tous les actants n'ont pas le même statut, toutes les relations ne sont pas identiques. Il y a en effet une hiérarchie entre les actants. La relation fondamentale est établie entre le prédicat et l'actant qui dénote l'entité sans laquelle on ne pourrait même pas commencer à imaginer la situation décrite. Cet actant fondamental est la branche dans les exemples suivants :

8 [3] a. La branche casse.

b. Julie casse la branche.

9 S'il n'y a rien qui se casse, la situation « casser » n'a pas eu lieu. Comme on le voit dans (3a), la proposition peut se réduire à la seule combinaison d'un prédicat avec son actant fondamental. Comme il ressort également des exemples, l'actant fondamental peut se réaliser comme le sujet d'un verbe intransitif ou comme l'objet d'un verbe transitif, parfois avec le même verbe, comme dans (3).

10 Cette idée d'un actant fondamental peut être quelque peu précisée à l'aide d'une classification des prédicats et le rôle que joue cet actant avec les différentes classes de verbes. La classification dont j'ai besoin tient compte de trois classes de verbes, deux simples et une complexe. On peut représenter ces trois classes de la façon suivante :

11 [4] Verbe d'état (simple) :

Verbe d'activité (simple) :

Verbe d'action (complexe) :

12 Si le verbe d'état dénote une situation stable, où il n'y a rien qui bouge (ex. rester) et le verbe d'activité dénote une situation instable, où il y a quelque chose qui bouge (ex. courir), le verbe d'action dénote une situation complexe où une activité (instabilité) aboutit à un état (stabilité) comme par exemple entrer. On reconnaît dans cette 
troisième classe complexe les verbes « téliques » ou encore « perfectifs » d'autres écoles et traditions. Pour notre propos ici, ce qui est important c'est que l'actant fondamental se définit comme le seul argument qui apparaît dans les deux parties de la représentation d'un verbe complexe (cf. Grimshaw 1992) :

[5]

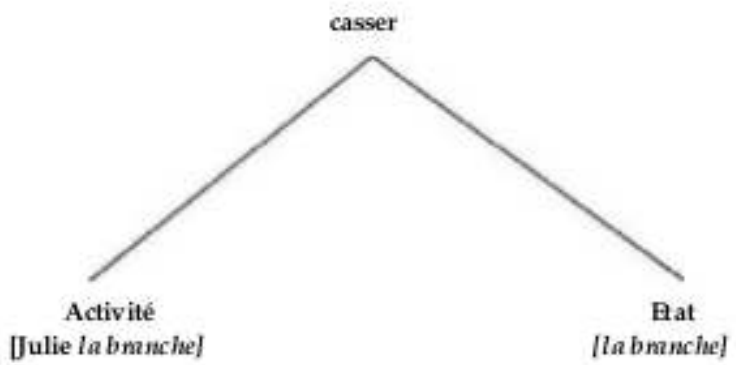

14 Comme il ressort du schéma, la représentation du verbe complexe exige deux composantes: une activité, où un agent extérieur (Julie) ou une force intérieure exercent un impact sur la branche, suivie d'un état où la branche est effectivement cassée.

En généralisant aux autres classes de verbes, on peut dire qu'avec les verbes d'état, l'actant fondamental est l'entité qui se trouve dans l'état dénoté :

16 [6] a. Le livre est sur la table.

b. Julie reste au lit.

c. Julie garde le secret.

17 Avec les verbes d'activité, l'actant fondamental est celui qui dénote l'entité qui bouge ou qui est soumis à l'activité décrite :

18 [7] a. Julie nage.

b. Julie secoue l'arbre.

c. Julie est en train de lire le journal.

19 Comme on le voit, l'actant fondamental est réalisé tantôt par un sujet, tantôt par un objet (voir à ce propos aussi Herslund 2001). Voici une première indication que la distinction entre proposition intransitive et proposition transitive sera d'une importance primordiale.

\subsection{Sujet et objet et leurs rôles respectifs}

Comme on vient de le voir, il y a parmi les actants un actant que j'ai nommé «fondamental»: dans la structure intransitive, c'est le sujet, dans la structure transitive, c'est l'objet. La distinction entre les fonctions respectives du sujet et de l'objet ressort le plus clairement de la structure transitive. Or, il apparait que l'objet n'a pas d'autre fonction que celle de réaliser l'actant fondamental, c'est-à-dire d'entrer dans une combinaison particulièrement intime avec le prédicat de sorte que les deux ensemble constituent ce qu'on peut appeler le fondement prédicatif (cf. Herslund \& Sorensen 1994). Cette combinaison intime se manifeste par plusieurs traits sémantiques et syntaxiques. Je ne citerai ici que deux de ces traits. Pour plus de détails, voir M. Herslund $(1988,1994,1997,2001)$ et M. Herslund \& F. Sorensen (1996). 
Avec les verbes polysèmes - en fait, tous les verbes sont plus ou moins polysèmes - c'est toujours l'objet ( = l'actant fondamental) qui détermine l'interprétation précise du verbe. Pour prendre un exemple classique, le verbe prendre:

[8]

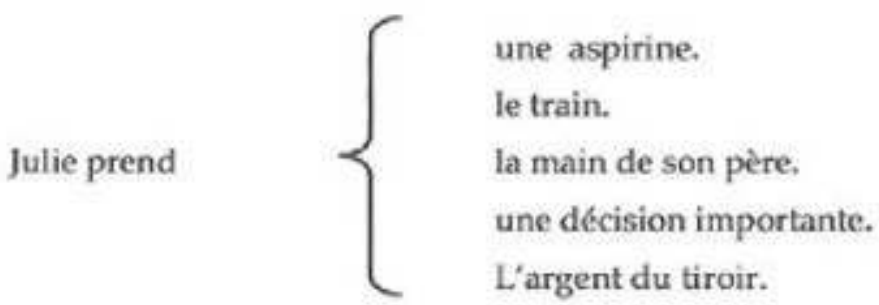

On voit que le sens de prendre, et par conséquent le type d'activité exécutée par le sujet, varie en fonction de l'objet choisi. Un changement de sujet, d'autre part, n'occasionnera jamais le moindre changement dans l'unité formée par le verbe et son objet, le fondement prédicatif : on observe ainsi une asymétrie fondamentale entre sujet et objet (cf. Marantz 1984).

L'autre trait que je mentionnerai ici est le fait que c'est l'objet, l'actant fondamental, qui, de façon régulière, se combine avec le participe passé des verbes transitifs pour former une construction attributive, un passif (processus) ou une construction stative (état), qui n'a pas reçu de nom particulier dans la tradition grammaticale :

[9] a. Les portes sont fermées (à six heures).

b. Les portes sont fermées (depuis hier matin).

La fonction du sujet est tout autre. Le sujet est l'argument avec lequel le prédicat constitue une proposition, c'est-à-dire une unité linguistique à laquelle on peut attribuer une valeur de vérité.

Nous avons par conséquent la représentation suivante des deux fonctions, objet et sujet :

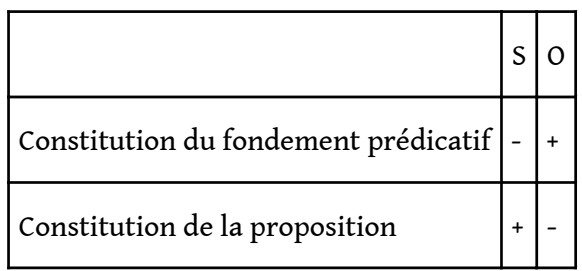

29 Cette analyse ne diffère pas fondamentalement des analyses en termes d'arguments internes et arguments externes (cf. par exemple Van Peteghem (à paraître), Tayalati 2005 et Asnes 2006).

\subsection{La distinction fondamentale entre proposition transitive et intransitive}

Le tableau (10) vaut pour la structure transitive. Mais comment décrire la structure intransitive ? Comme parfois le sujet est le seul actant, il s'ensuit que le sujet en ce cas assure aussi la fonction d'actant fondamental, et que les deux fonctions identifiées se 
confondent dans un seul et même actant. La structure intransitive comporte donc les deux mêmes fonctions que la structure transitive, mais le sujet intransitif est à la fois l'argument qui constitue le fondement prédicatif et l'argument qui constitue la proposition. Il remplit les mêmes fonctions que celles qui, dans la structure transitive, sont distribuées sur l'objet et le sujet respectivement. Nous pouvons donc compléter le schéma [10]:

\begin{tabular}{|l|l|l|l|}
\hline & St & Si & O \\
\hline Constitution du fondement prédicatif & - & + & + \\
\hline Constitution de la proposition & + & + & - \\
\hline
\end{tabular}

31 A l'intérieur de la classe des verbes intransitifs, il faut pourtant tenir compte d'une distinction entre verbes inaccusatifs (ou "ergatifs ») et verbes inergatifs (cf. Herslund 1990 \& 2001 et toute la littérature importante consacrée à ce problème, cf. par exemple Levin \& Rappaport Hovav 1995). Quand, par la suite, je parle de structures intransitives, il s'agit uniquement de structures contenant des verbes inaccusatifs, dont le sujet a les propriétés d'objet décrites ci-dessus.

32 Le parallélisme entre le sujet intransitif (inaccusatif) et l'objet est mis en relief par le fait que ce sujet a les mêmes propriétés que l'objet illustrées sous [8] et [9]), à savoir la détermination du sens exact à attribuer à un verbe polysème :

33 [11]

\begin{tabular}{|c|c|c|}
\hline Le train & & du tunnel. \\
\hline L'air & & du ballon. \\
\hline Le clou & sort & du mur. \\
\hline Julie & & avec ses amies. \\
\hline
\end{tabular}

34 et la capacité de former une construction attributive avec le participe passé de son verbe, [12], construction qui ne se confond pas avec le passé composé formé avec l'auxiliaire être :

35 [12] a. Julie est sortie (à trois heures).

b. Julie est sortie (depuis trois heures).

\subsection{La notion d'adjet}

Avec les deux actants centraux, sujet et objet, on pourrait dire qu'il n'y a plus de place autour du verbe, que sa valence est saturée. Selon l'analyse proposée ci-dessus, la structure transitive peut être représentée comme sous [13], où le '+' signale l'actant fondamental et le fondement prédicatif : 


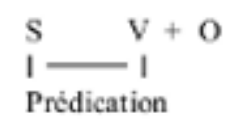

b. Intransitif

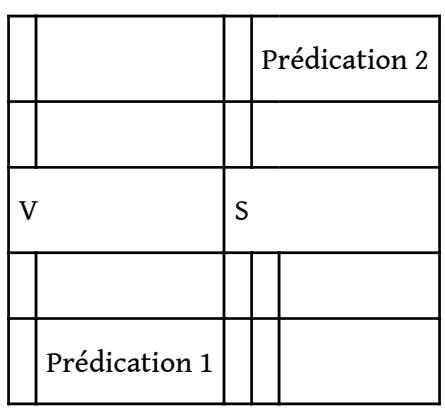
comme sous [14]:

[14]

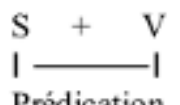

Prédication

[15] Il faut joindre l'utile à l'agréable.

[16]a. Transitif

Prédication 2

et la structure intransitive, où le sujet a une fonction double comme signalé plus haut,

La seule possibilité pour l'introduction d'un troisième actant est en effet au moyen d'une prédication seconde. Cette prédication seconde est toujours construite selon les mêmes lignes : c'est toujours l'actant fondamental - $\mathrm{O}$ ou $\mathrm{S}$ - qui se voit ajouter un complément, prépositionnel ou attributif. C'est ce complément que nous avons appelé, avec notre regretté collègue et ami, Finn Sorensen, adjet (cf. Herslund \& Sorensen 1994 \& 1996) - pour construire un terme inusité qui fait écho à «objet » et « sujet ». Comme illustration préalable de l'adjet, voici une phrase modèle avec le verbe joindre, qui illustre aussi le fondement sémantique de toutes les constructions avec un adjet à préposition à, à savoir le placement - plus ou moins concret - d'une entité par rapport à une autre entité, qui de ce fait devient un lieu (voir plus bas) :

Les structures élargies, transitive et intransitive, auront les représentations suivantes :

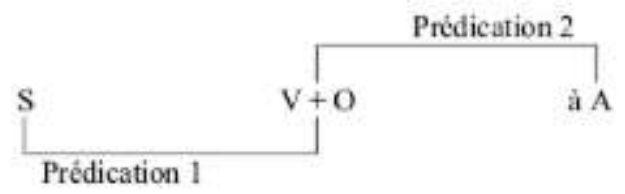

Il faut aussi insister sur le fait, qui étaie l'analyse proposée, qu'une structure trivalente dans laquelle une telle prédication seconde est établie entre le sujet (transitif) et un adjet, semble inexistante : un sujet transitif n'est jamais l'actant fondamental.

A la lumière de cette analyse, le rôle de la préposition devient clair aussi : c'est celui d'établir, au niveau syntaxique, cette prédication seconde (cf. Cadiot 2000). Nous avons 
donc de façon régulière une proposition contenant deux prédications, la première construite par un verbe, la seconde par une préposition :

47 [17]

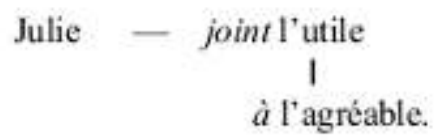

Comme il fallait s'y attendre, les deux prédications se confondent dans la structure intransitive, dû à la fonction double du sujet intransitif, qui, étant l'actant fondamental, est le sujet à la fois de la prédication première et de la prédication seconde :

[18]

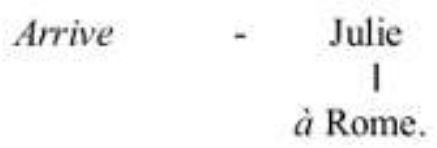

L'adjet se manifeste donc comme un complément prépositionnel (je reviendrai plus loin sur sa manifestation sous forme d'attribut adjectival) dont le sens varie entre l'interprétation concrètement locative et une interprétation plus abstraite où le rôle de l'entité dénotée par le syntagme adjet est plutôt celui d'un récipient ou d'un destinataire : il s'agit ici du datif (cf. Herslund 1988a, Melis 1996). La base locative des verbes qui se construisent avec un adjet avec la préposition à peut être illustrée à l'aide du verbe laisser :

[19] a. Julie a laissé son vélo à la gare.

b. Julie a laissé son vélo à son frère.

Dans [19]a, on a l'interprétation locative concrète, [19]b illustre, avec le même verbe, le sens plus abstrait qu'on associe à un datif.

Si le moule syntaxique est donc toujours le même, le fondement sémantique de la prédication seconde l'est aussi : il s'agit toujours d'une localisation de (l'entité dénotée par) l'actant fondamental par rapport à un lieu. Qu'est-ce qu'un lieu? Un « lieu » n'est pas un concept primitif, c'est un concept construit : une ville, une maison, un tiroir, etc. ne sont pas d'emblée des lieux, ce sont des espaces. Mais quand il y a une entité qui se situe par rapport à eux, ils se transforment en lieux : c'est grâce à la présence d'un argument - l'entité qui est située - qu'un espace, où n'importe quel objet, devient un lieu (cf. Guillet \& Leclère 1992, Herslund \& Baron 1999 \& 2001). C'est cette "prédication» conceptuelle (ou cognitive ?) qui constitue la base de la prédication seconde sémantique, qui se réalise sous la forme grammaticale d'une construction prépositionnelle « adjet».

54 Sémantiquement parlant, la construction à adjet peut être décrite par une paraphrase où le verbe (trivalent) est décomposé en deux prédicats, un prédicat causatif qui désigne la prédication première, et un prédicat locatif correspondant à la prédication seconde, exprimée grammaticalement par la préposition :

[20] a. Julie laisse son vélo à la gare.

b. JULIE CAUSER [SON VÉLO ÊTRE À LA GARE]

Comme on vient de le voir [sous (19)], le même prédicat sélectionne tantôt un adjet locatif, tantôt un adjet datif. Sémantiquement, en fait, le datif est beaucoup plus qu'un 
locatif «banal », tout en possédant pourtant certains traits qui le rapprochent du locatif. On peut dire, en effet, que le datif est un locatif abstrait ou métaphorique en ce sens qu'on y retrouve les mêmes ingrédients qu'avec le locatif, à savoir un lieu et un argument de ce lieu. Mais le rôle qu'on attribue communément au datif est plutôt celui d'un récipient (destinataire), et ce qui distingue un récipient d'un lieu, c'est le fait que celui-là participe de façon active à un échange et ne constitue pas simplement le support inerte de quelque chose. Ce côté «actif» du datif peut être représenté en renversant simplement les termes de la prédication seconde dans la paraphrase sémantique : de complément de lieu, l'argument correspondant à l'adjet devient le sujet d'un verbe être renversé, c'est-à-dire avoir, dans la prédication seconde. D'où la paraphrase suivante :

$57\left[20^{\prime}\right]$ a. Julie laisse son vélo à son frère.

b. JULIE CAUSER [SON FRÈRE AVOIR SON VELO]

58 Entre les deux extrêmes, l'adjet locatif et l'adjet datif, il y a une zone de constructions avec la préposition à qui n'expriment ni la localisation concrète du locatif, ni la localisation abstraite du datif, mais une sorte de localisation abstraite sans le renversement des arguments qui caractérise par ailleurs la paraphrase du datif. Cet adjet « neutre » est le plus souvent réalisé par un nom abstrait, un nom déverbal ou un syntagme infinitif :

59 [21] a. Locatif : Julie amène son frère à la gare.

b. Neutre : Julie amène son frère à changer d'avis.

c. Datif : Julie amène son frère au roi. classifier les verbes français, transitifs et intransitifs, en quatre groupes majeurs, mais je ne peux pas entrer dans les détails d'une telle classification ici (voir Herslund 1988a). Syntaxiquement, les trois types d'adjets qui se construisent avec la préposition à, se distinguent, entre autres (cf. Herslund 1988a), par leurs choix de pronoms clitiques (y pour l'adjet locatif et neutre, lui pour l'adjet datif) et par le fait que la préposition, qui exprime la prédication seconde, peut varier avec l'adjet locatif, mais est toujours à avec l'adjet neutre et datif :

63 [23]

\begin{tabular}{|c|c|c|}
\hline & itique & Préposition \\
\hline \multirow{2}{*}{$\begin{array}{l}\text { Locatif } \\
\text { Neutre }\end{array}$} & \multirow[b]{2}{*}{ y } & $\{\mathrm{a}+\mathrm{duns}+$ chez + sur + sous $\ldots\}$ \\
\hline & & \multirow[t]{2}{*}{ à } \\
\hline Datif & lui & \\
\hline
\end{tabular}




\subsection{L'attribut}

64 Nous arrivons finalement à la dernière manifestation de l'adjet, à savoir l'attribut. Comme les autres manifestations qu'on vient de voir, l'adjet attribut se trouve en construction intransitive (attribut du sujet) et transitive (attribut de l'objet). Comme prévu, il s'agit dans les deux cas d'une prédication seconde axée sur l'actant fondamental: il n'y a pas d'attribut du sujet transitif - ce qui serait pourtant «logiquement» possible! De nouveau, c'est ici aussi une préposition qui réalise la prédication seconde, sauf pour la classe de mots qu'on associe plus particulièrement à la fonction d'attribut, les adjectifs. Avec eux, c'est l'accord qui signale la présence de la prédication seconde :

[24] a. Julie est en colère.

b. Julie est furieuse.

66 [25] a. Cette décision [ on ] a mis Julie en colère.

b. Cette décision a rendu Julie furieuse.

\section{Conclusion}

67 Le but du présent article a été de démontrer, à l'aide d'une description de la valence verbale et les conséquences syntaxiques d'une telle analyse, que le rôle de la préposition n'est pas celui d'un simple élément de liaison, mais que la préposition joue en effet souvent un rôle de prédicat (ou de "prédicatoïde ») dans une prédication seconde. L'idée sous-jacente à la description valentielle présentée est en effet que la structure de la phrase n'est pas «plate » ou simplement «linéaire ", comme le font croire par exemple les stemmas de L. Tesnière (1959). La structure de la phrase est construite autour d'une hiérarchisation des actants, qui jouent chacun un rôle déterminé dans cette construction : il y a d'abord la construction d'un fondement prédicatif (prédicat + actant fondamental) ; ensuite la construction d'une proposition (sujet + fondement prédicatif); et enfin, parfois la construction d'une prédication seconde à l'aide d'une structure prépositionnelle (actant fondamental + adjet). Cette description, doublée d'une distinction basique entre phrase transitive et phrase intransitive, prévoit en effet l'existence de seulement quatre types de phrases de base en français :

\begin{tabular}{|l|l|l|}
\hline & + Adjet & - Adjet \\
\hline Transitif & $\mathrm{SV}+\mathrm{O} \mathrm{A}$ & $\mathrm{SV}+0$ \\
\hline Intransitif & $\mathrm{S}+\mathrm{V} \mathrm{A}$ & $\mathrm{S}+\mathrm{V}$ \\
\hline
\end{tabular}

Ces quatre types sont illustrés par les exemples suivants :

[26'] a. Julie attribue de l'importance à ces propos.

b. Julie apprécie ces propos.

c. Julie arrive à sa conclusion.

d. Julie disparait. 
71 Pour des raisons d'espace, j'ai dû limiter la discussion ici aux adjets construits autour de la préposition à, laissant ainsi de côté le groupe important des adjets avec de, qui souvent forment des structures en image de miroir par rapport aux constructions avec à, cf. par ex. :

[27] a. Julie demande à Pierre de fermer la porte.

b. Julie prie Pierre de fermer la porte.

Si dans [27]a on a une construction avec à Pierre adjet et le syntagme infinitif [de fermer la porte] comme objet, dans [27]b, c'est Pierre qui est objet et le syntagme infinitif [fermer la porte] un adjet introduit par la préposition de. Des contrastes comme ceux de [27] amènent certains (par exemple, Dryer 1986) à voir dans la structure de [27]b une sorte d' « antidatif », ce qui, à mon avis, se justifie pleinement dans le cadre esquissé ici. Mais là, ce serait aborder une autre discussion.

\section{RÉSUMÉS}

Michael Herslund démontre la pertinence de la notion d' « adjet » (terme forgé sur le modèle de "sujet» et "objet») qui prend place dans une théorie globalement empruntée à Tesnière ( "valence », « prédicat », " actant », « circonstant », ...). La distinction établie, syntaxiquement et sémantiquement, entre construction transitive et construction intransitive, sujet et objet, montre qu'il y a place pour un troisième actant mais via une prédication seconde - fonction dénommée « adjet » assurée par exemple par à l'agréable dans joindre l'utile à l'agréable.

\section{AUTEUR}

\section{MICHAEL HERSLUND}

Ecole des Hautes Etudes Commerciales de Copenhague 